\title{
Liver stiffness-spleen size-to-platelet ratio risk score detects esophageal varices in chronic liver disease
}

\author{
Soichiro Shibata ${ }^{\dagger}$, Satoru Joshita ${ }^{\dagger}$, Takeji Umemura $^{*}$ (1), Tomoo Yamazaki, Naoyuki Fujimori, Yuki Ichikawa, \\ Michiharu Komatsu, Akihiro Matsumoto and Eiji Tanaka
}

\begin{abstract}
Background: Noninvasive markers are needed to identify esophageal varices (EV) in patients with chronic liver disease (CLD). Recently, liver stiffness (LS)-spleen size-to-platelet ratio risk score (LSPS) has been shown to predict EV in patients with chronic hepatitis C. The aim of this study was to validate the clinical value of LSPS for EV detection and identification of high risk EV in Japanese patients with CLD.

Methods: A total of 230 patients with CLD who had undergone endoscopy, LS measurement, and ultrasonography between 2013 and 2015 were enrolled. The relationships between clinical data and LSPS were compared with those for other noninvasive markers (aspartate aminotransferase-to-platelet ratio, FIB-4 index, and platelet-to-spleen ratio), along with platelet count, spleen size, and LS. Diagnostic and prognostic abilities were assessed by the area under the receiver operating characteristic curve (AUC) and multivariate logistic regression.

Results: LSPS correlated significantly with EV grade $(P<0.001)$ and was superior to the other noninvasive indices for determination of EV and high risk EV. Furthermore, LSPS was independently associated with the presence of EV $(P<0.001)$ and elevated EV risk $(P=0.013)$ by multivariate logistic regression analysis. The optimal cutoff values of LSPS for EV and high risk EV were 1.1 and 2.2, respectively, at which AUC, negative predictive value, and accuracy were 0.821 [95 \% confidence interval (Cl) 0.743-0.899], 91.9, and $84.3 \%$ and 0.859 (95 \% Cl 0.736-0.981), 95.5, and 76.9\%, respectively.
\end{abstract}

Conclusions: LSPS represents a useful, noninvasive, accurate method to detect EV and a high EV risk in Japanese patients with CLD.

Keywords: LSPS, Transient elastography, CLD, Esophageal varices

\section{Background}

Esophageal varices (EV) are the most relevant portosystemic collaterals resulting from clinically significant portal hypertension, for which the presence of $\mathrm{EV}$ is an independent predictor of mortality (D'Amico et al. 2006). As acute variceal bleeding is a major complication of cirrhosis, patients with newly diagnosed cirrhosis in chronic liver disease (CLD) are advised to undergo endoscopic

\footnotetext{
${ }^{*}$ Correspondence: tumemura@shinshu-u.ac.jp

tSoichiro Shibata and Satoru Joshita contributed equally to this work Division of Hepatology and Gastroenterology, Department of Medicine, Shinshu University School of Medicine, 3-1-1 Asahi, Matsumoto 390-8621, Japan
}

screening for EV (de Franchis and Baveno 2010). However, endoscopy is an invasive and unpleasant procedure that carries rare, but serious, complications. Thus, simple, noninvasive, accurate tests are needed to predict EV in CLD. The liver stiffness (LS)-spleen size-to-platelet ratio score (LSPS), which is a combination of 3 simple examination methods (LS, spleen size, and platelet count) has been found to predict $\mathrm{EV}$ and high risk EV in patients with compensated cirrhosis (Berzigotti et al. 2013; Kim et al. 2010). We also recently reported that LSPS could identify EV in Japanese patients with chronic hepatitis $\mathrm{C}$ virus (HCV) infection (Shibata et al. 2015). Since this method has not yet been validated for other etiologies 
of CLD in Japan, the present investigation evaluated the ability of LSPS to predict the presence of EV and high risk EV in Japanese patients with CLD.

\section{Methods Subjects}

A total of 835 consecutive patients with CLD who were seen at Shinshu University Hospital (Matsumoto, Japan) between April 2013 and December 2015 and evaluated by endoscopy, LS measurement, and ultrasonography within an interval of 6 months and without a history of variceal bleeding or ascites were recruited. Exclusion criteria were as follows: (1) fewer than 10 LSM measurements, (2) IQR of greater than $30 \%$, (3) receiving splenectomy, (4) lack of laboratory data, (5) lack of clinical data, and (6) HCV infection [the data on which have already been published (Shibata et al. 2015)], as presented in Fig. 1. Ultimately, a total of 230 patients with CLD were enrolled in this retrospective, cross-sectional study. The diagnosis of CLD was based on the following disease criteria: hepatitis B surface antigen and hepatitis B virus (HBV) DNA in patients positive for hepatitis $B$ surface antigen were evaluated to identify persistent $\mathrm{HBV}$ infection (Umemura et al. 2008). Autoimmune hepatitis (Umemura et al. 2007) and primary biliary cirrhosis (Umemura et al. 2012) were diagnosed by histological examination and serological

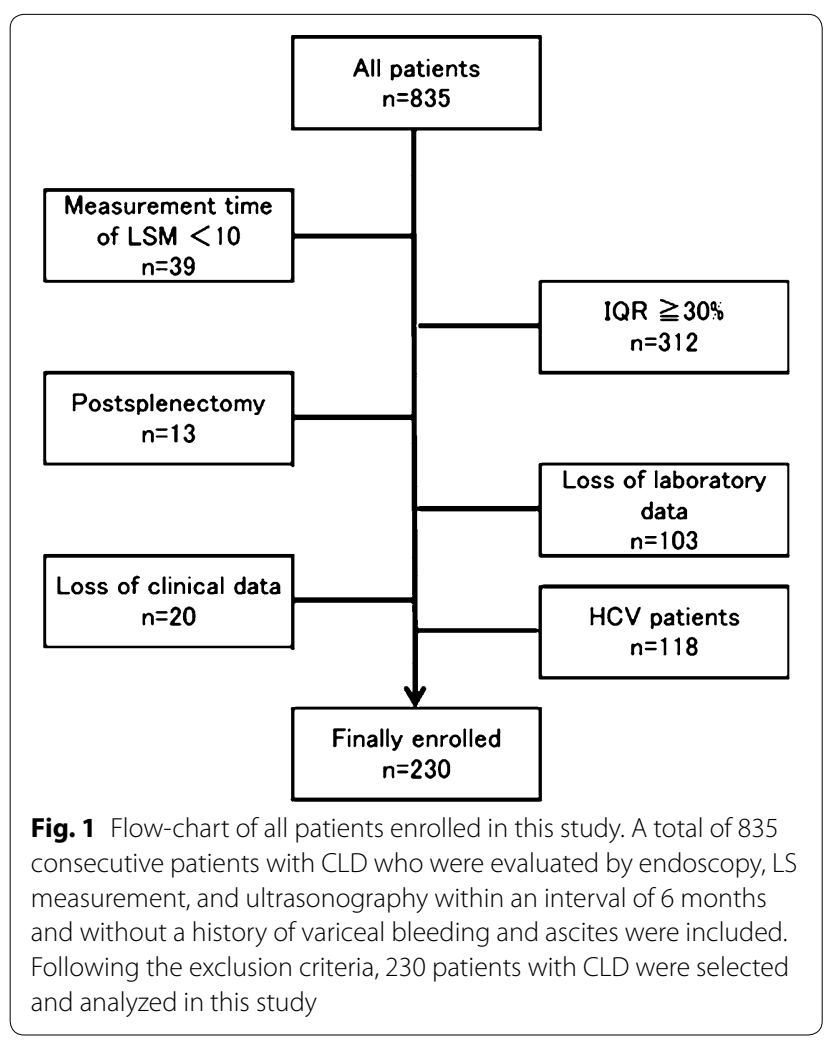

testing, as previously reported. Alcoholic liver disease and non-alcoholic fatty liver disease (NAFLD) were determined using conventional methods. Non-alcoholic steatohepatitis (NASH) was diagnosed by histological examination (Kleiner et al. 2005) (Additional file 1: Figure S1).

\section{Research ethics considerations}

This study was conducted in accordance with the principles of the 1975 Declaration of Helsinki. The protocol of this investigation was reviewed and approved by the Shinsu University ethics committee (number 3021). Informed consent was obtained from all participants.

\section{Laboratory testing}

All laboratory data were obtained on the same day as transient elastography (TE) scanning. Alanine aminotransferase, aspartate aminotransferase, and other relevant biochemical tests were performed using standard methods. Four surrogate blood indices of liver fibrosis and EV prediction were also assessed at enrollment according to published formulae: aspartate aminotransferase-to-platelet ratio index (APRI) (Wai et al. 2003) and FIB-4 index (Sterling et al. 2006) were calculated as: (AST/upper limit of normal; $40 \mathrm{IU} / \mathrm{l}) \times[100 /$ platelet count $\left.\left(10^{9} / \mathrm{l}\right)\right]$ and [age (years) $\times$ AST (IU/l)]/[platelet count $\left.\left(10^{9} / \mathrm{l}\right) \times \operatorname{ALT}(\mathrm{IU} / \mathrm{l})^{1 / 2}\right]$, respectively. The plateletto-spleen ratio (Giannini et al. 2003) and LSPS (Berzigotti et al. 2013; Kim et al. 2010) formulae were as follows: platelet count/spleen diameter and LS value $\times$ spleen diameter/ platelet count, respectively.

\section{Ultrasonography (US) and TE}

US and TE were performed on the same day following overnight fasting. Spleen size was measured on US images before TE as spleen bipolar diameter (i.e., crossing the spleen hilium) with a convex probe. The experienced US operators were unaware of the patients' clinical details.

TE was determined with a FibroScan device (FibroScan502, Echosens, Paris, France) using a $50 \mathrm{~Hz}$ standard M probe, as previously described (Sandrin et al. 2003). All patients were assessed using a $3.5 \mathrm{MHz}$ standard $\mathrm{M}$ probe (EUB-8500, Hitachi Medical Co., Ltd., Japan). LS was determined as the median value of 10 measurements and expressed in kilopascals $(\mathrm{kPa})$.

\section{Endoscopy for EV}

Two experienced endoscopists who were blinded to the LS results performed all endoscopic examinations. EV staging was classified as none (no veins above the esophageal mucosal surface; F0), small (minimally elevated veins above the esophageal mucosal surface; F1), medium (large tortuous veins occupying $<1 / 3$ of the lumen; F2), or 
large (large coil-shaped veins occupying $\geq 1 / 3$ of lumen; F3) according to standard criteria published by the Japan Society for Portal Hypertension (Beppu et al. 1981). The high risk EV group included all patients with F2/F3 EV or those having F1 EV with red color signs.

\section{Statistical analysis}

Categorical variables were compared using the Chi squared or Fisher's exact tests, as appropriate. Continuous variables were assessed by the Mann-Whitney $U$ or Kruskal-Wallis tests, and abnormally distributed variables were determined by the Shapiro-Wilk test. Diagnostic accuracy was calculated using receiver operating characteristic (ROC) curve analysis in terms of sensitivity, specificity, positive predictive value (PPV), negative predictive value (NPV), and area under the ROC curve (AUC). Cutoff values were identified by the Youden index, and the nearest clinically applicable value to the cutoff was considered as the optimal cutoff value for clinical convenience. Multivariate forward stepwise logistic regression analysis was employed to identify independent factors predictive of the absence or presence of EV and high risk EV. Comparisons of paired AUCs and $95 \%$ confidence intervals (CIs) were carried out using the nonparametric Delong test. A $P<0.05$ was considered to be statistically significant. Statistical analyses were performed using IBM SPSS Statistics ver. 21.0 (IBM, Chicago, IL) and StatFlex ver. 6.0 (Artech Co., Ltd. Osaka, Japan) software.

\section{Results}

\section{Baseline clinical characteristics of patients}

The baseline characteristics of the 230 patients are summarized in Table 1. Median age was 63 years and male incidence was $49 \%$. The main etiology of CLD was NAFLD/NASH (25\%), followed next by HBV (20\%), primary biliary cirrhosis (18\%), alcoholic liver disease (10\%), autoimmune hepatitis (7\%), and others (20\%), such as cryptogenic cirrhosis, Budd-Chiari syndrome, hemochromatosis, primary sclerosing cholangitis, and drug-induced liver injury. One hundred and ninetyone patients had no EV, 31 had F1 EV (2 with red color signs), 7 had F2 (4 with red color signs) and 1 had F3 and red color signs. Overall, 29 patients belonged to the low risk EV group and 10 belonged to the high risk EV group. We conducted an additional sub-analysis in terms of etiology, which included alcoholic LD $(n=23)$, and found that these patients displayed a significantly higher LS than did others $(P<0.001$ as compared with each etiology). However, since the number of alcoholic LD subjects was too small to make a definitive conclusion, we conducted the study to include all etiologies apart from $\mathrm{HCV}$.
Table 1 Baseline characteristics of 230 CLD patients

\begin{tabular}{lc}
\hline Characteristic & Total $(\mathbf{n}=\mathbf{2 3 0})$ \\
\hline Age (years) & $63(10-90)$ \\
Male gender & $113(49)$ \\
Body mass index $\left(\mathrm{kg} / \mathrm{m}^{2}\right)$ & $22.7(11.9-41.1)$ \\
Etiology & \\
NAFLD/NASH & $57(25)$ \\
HBV & $47(20)$ \\
PBC & $42(18)$ \\
ALD & $23(10)$ \\
AlH & $15(7)$ \\
Other & $46(20)$ \\
Platelet count $\left(\times 10^{9} / \mathrm{l}\right)$ & $182(14-713)$ \\
AST (IU/l) & $30(6-1261)$ \\
ALT (IU/l) & $28(6-1246)$ \\
Albumin (g/dl) & $4.1(1.4-4.9)$ \\
T.Bil (mg/dl) & $0.8(0.2-24.2)$ \\
PT\% & $92(24-128)$ \\
LS (kPa) & $5.9(1.5-75.0)$ \\
Spleen size (cm) & $8.9(3.9-24.0)$ \\
LSPS & $0.3(0.0-20.0)$ \\
APRI & $0.5(0.1-33.7)$ \\
FIB-4 & $1.9(0.1-25.5)$ \\
Platelet-to-spleen ratio & $2091(166-7809)$ \\
\hline
\end{tabular}

Values are expressed as median (range, minimum-maximum) or $\mathrm{n}(\%)$

\section{Diagnostic performance of noninvasive methods for EV}

The clinical characteristics of patients with and without EV are shown in Table 2. Total bilirubin was significantly higher in patients with EV than in those without, while platelet count, albumin, and PT\% were significantly lower. All noninvasive markers (LSPS, APRI, FIB-4, and platelet-to-spleen ratio) were significantly associated with EV (all $P<0.001$ ). Multivariate analysis demonstrated that LSPS [odds ratio (OR) 2.441, $95 \%$ CI 1.747 3.413; $P<0.001$ ] was independently associated with the presence of EV. As shown in Fig. 2, median LSPS values were 0.2 (IQR 0.1-0.5), 1.0 (IQR 0.3-2.9), and 5.5 (IQR 2.4-12.5) in the no EV, low risk EV, and high risk EV groups, respectively. LSPS values were significantly correlated with EV severity $(P<0.001$, Kruskal-Wallis test).

We next performed ROC curve analysis to determine the predictive accuracy of the noninvasive parameters for EV in CLD. The values for AUC, optimal cutoff value, sensitivity, specificity, PPV, NPV, and accuracy for the presence of EV are listed in Table 3. AUCs were 0.821 for LSPS, 0.807 for platelet-to-spleen ratio, 0.800 for platelet count, 0.779 for FIB- 4 index, 0.775 for spleen size, 0.765 for LS, and 0.749 for APRI. Although LSPS had the highest discrimination for $\mathrm{EV}$, there were no significant differences between the AUC of LSPS and those for 
Table 2 Comparison between patients with and without EV

\begin{tabular}{|c|c|c|c|}
\hline Characteristic & $\mathrm{EV}(+)(n=39)$ & $\operatorname{EV}(-)(n=191)$ & $P$ value \\
\hline Age (years) & $61(13-90)$ & $63(10-87)$ & 0.553 \\
\hline Male gender & $25(64)$ & $88(46)$ & 0.040 \\
\hline Body mass index $\left(\mathrm{kg} / \mathrm{m}^{2}\right)$ & $24.5(17.3-41.1)$ & $22.7(11.9-41.0)$ & 0.037 \\
\hline \multicolumn{4}{|l|}{ Etiology } \\
\hline NAFLD/NASH & $7(18)$ & $50(26)$ & 0.378 \\
\hline HBV & $10(26)$ & $37(19)$ & 0.377 \\
\hline PBC & $5(13)$ & $37(19)$ & 0.461 \\
\hline ALD & $10(26)$ & $13(7)$ & 0.001 \\
\hline AlH & $0(0)$ & $15(8)$ & 0.146 \\
\hline Other & $7(18)$ & $39(20)$ & 0.895 \\
\hline Platelet count $\left(\times 10^{9} / \mathrm{l}\right)$ & $99(34-683)$ & $197(14-713)$ & 0.013 \\
\hline AST (IU/I) & $33(15-146)$ & $29(6-1261)$ & 0.194 \\
\hline ALT (IU/I) & $25(8-123)$ & $28(6-1246)$ & 0.196 \\
\hline Albumin (g/dl) & $3.7(2.7-4.6)$ & $4.2(1.4-4.9)$ & 0.005 \\
\hline T.Bil (mg/dl) & $1.2(0.4-7.5)$ & $0.7(0.2-24.2)$ & $<0.001$ \\
\hline РТ\% & $74(27-95)$ & $95(24-128)$ & $<0.001$ \\
\hline LS (kPa) & $10.0(3.5-75.0)$ & $5.3(1.5-75.0)$ & $<0.001$ \\
\hline Spleen size $(\mathrm{cm})$ & $11.4(6.1-24.0)$ & $8.6(3.9-14.8)$ & $<0.001$ \\
\hline LSPS & $1.7(0.1-20.0)$ & $0.2(0.0-6.4)$ & $<0.001$ \\
\hline APRI & $1.4(0.1-4.8)$ & $0.5(0.1-33.7)$ & $<0.001$ \\
\hline FIB-4 & $5.7(0.2-12.0)$ & $1.8(0.1-25.5)$ & $<0.001$ \\
\hline Platelet-to-spleen ratio & $770(220-5991)$ & 2201 (166-7809) & $<0.001$ \\
\hline
\end{tabular}

Values are expressed as median (range, minimum-maximum) or $\mathrm{n}(\%)$

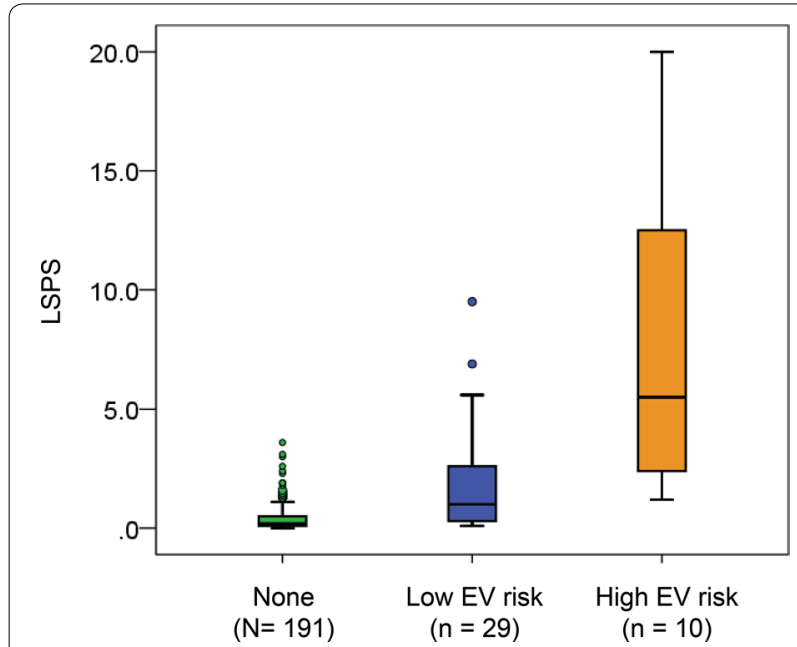

Fig. 2 LSPS detection of the presence and high risk EV. Boxes represent the IQR of the data. The lines across the boxes indicate median values. The hash marks above and below the boxes indicate the 90th and 10th percentiles for each group, respectively. Open circles indicate outliers

platelet-to-spleen ratio, platelet count, FIB-4, spleen size, LS, or APRI. An LSPS cutoff value of 1.1 yielded a sensitivity of $61.5 \%$, specificity of $89.0 \%$, PPV of $53.3 \%$, NPV of $91.9 \%$, and accuracy of $84.3 \%$ (Table 3 ).
Prediction of high risk EV in 39 patients with EV

Among the 39 patients complicated with EV, total bilirubin was significantly higher in patients with high risk EV than in those without, while platelet count and PT\% were significantly lower. Among the noninvasive markers, patients with high risk EV had significantly higher LSPS, APRI, and FIB-4 and lower platelet-to-spleen ratio as compared with patients with low risk EV (Table 4). Multivariate analysis disclosed that LSPS only (OR 1.456, $95 \%$ CI 1.083-1.957; $P=0.013$ ) was independently associated with a high risk of EV in CLD.

The performance of noninvasive parameters for identifying high risk EV, including AUC, optimal cutoff value, sensitivity, specificity, PPV, NPV, and accuracy, is summarized in Table 5. Calculated AUCs were 0.859 for LSPS, 0.833 for platelet count, 0.817 for plateletto-spleen ratio, 0.807 for LS, 0.762 for APRI, and 0.716 for FIB-4. Although LSPS had the best discrimination for high risk EV, there were no significant differences between the AUC of LSPS and those of platelet count, platelet-to-spleen ratio, LS, or APRI. The optimal LSPS cutoff value of 2.2 provided a sensitivity of $90.0 \%$, specificity of $72.4 \%$, PPV of $52.9 \%$, NPV of $92.5 \%$, and accuracy of $76.9 \%$ (Table 5 ).

\section{Discussion}

Although LSPS has shown promise as a predictive marker of EV and/or high risk EV (Berzigotti et al. 2013; Kim et al. 2010; Shibata et al. 2015), additional trials are needed to validate its clinical utility. The present study confirmed the diagnostic accuracy of LSPS for detecting EV and high risk EV in patients with CLD of various etiologies. Moreover, LSPS was well correlated with EV grade $(P<0.001)$. The diagnostic accuracy and AUC of LSPS for identifying EV were $84.3 \%$ and 0.821 , respectively, and multivariate analysis revealed that LSPS had the highest performance in identifying EV in CLD. Hence, the current study on patients with CLD of various etiologies validated the usefulness of LSPS found in our prior report on chronic HCV infection (Shibata et al. 2015). The EV cutoff value was low in this study, indicating that those patients who displayed a lower value were presumed not to be complicated with EV based on the NPV. However, clinicians should recommend further endoscopic evaluation for CLD patients scoring greater than the LSPS cutoff value to evaluate for $\mathrm{EV}$, even though endoscopy is an unpleasant procedure that carries rare, but serious, complications.

Simple, reliable, noninvasive methods are sought to better identify high-risk cases of F2/F3 EV or F1 EV with red color signs prior to endoscopy and thus avoid variceal bleeding. The diagnostic accuracy of LSPS as determined by the AUC in the current study was similar to that of earlier published data (Kim et al. 2010; Shibata et al. 2015). 
Table 3 Performance of noninvasive parameters for identifying EV

\begin{tabular}{|c|c|c|c|c|c|c|c|c|c|}
\hline Characteristic & Cutoff & AUC (95\% Cl) & $\begin{array}{l}\text { Sensitivity } \\
(\%)\end{array}$ & $\begin{array}{l}\text { Specificity } \\
(\%)\end{array}$ & PPV (\%) & $\begin{array}{l}\text { NPV } \\
(\%)\end{array}$ & $\begin{array}{l}\text { Positive } \\
\text { Likelihood } \\
\text { ratio }\end{array}$ & $\begin{array}{l}\text { Negative } \\
\text { Likelihood } \\
\text { ratio }\end{array}$ & $\begin{array}{l}\text { Accuracy } \\
(\%)\end{array}$ \\
\hline LSPS & 1.1 & $0.821(0.743-0.899)$ & 61.5 & 89.0 & 53.3 & 91.9 & 5.60 & 0.43 & 84.3 \\
\hline Platelet count $\left(\times 10^{9} / \mathrm{I}\right)$ & 14.3 & $0.800(0.708-0.892)$ & 79.5 & 79.6 & 44.3 & 95.0 & 3.89 & 0.26 & 79.6 \\
\hline Platelet-to-spleen ratio & 1330 & $0.807(0.712-0.901)$ & 76.9 & 84.3 & 50.0 & 94.7 & 4.90 & 0.27 & 83.0 \\
\hline Spleen size (cm) & 9.8 & $0.775(0.697-0.852)$ & 74.4 & 79.1 & 42.0 & 93.8 & 3.55 & 0.32 & 78.3 \\
\hline $\mathrm{LS}(\mathrm{kPa})$ & 6.1 & $0.765(0.688-0.843)$ & 84.6 & 61.3 & 30.8 & 95.1 & 2.18 & 0.25 & 65.2 \\
\hline APRI & 1.2 & $0.749(0.655-0.844)$ & 59.0 & 84.8 & 44.2 & 91.0 & 3.88 & 0.48 & 80.4 \\
\hline FIB-4 index & 4.1 & 0.779 (0.690-0.868) & 61.5 & 89.5 & 54.5 & 91.9 & 5.88 & 0.43 & 84.8 \\
\hline
\end{tabular}

Table 4 Characteristics of patients with and without high risk EV

\begin{tabular}{|c|c|c|c|}
\hline Characteristic & Low risk $(n=29)$ & High risk $(n=10)$ & $P$ value \\
\hline Age (years) & 65 (13-90) & $54(14-74)$ & 0.145 \\
\hline Male & $17(59)$ & $8(80)$ & 0.279 \\
\hline Body mass index & $24.3(17.3-41.1)$ & $25.0(18.5-30.2)$ & 0.530 \\
\hline \multicolumn{4}{|l|}{ Etiology } \\
\hline NAFLD/NASH & $4(14)$ & $3(30)$ & 0.500 \\
\hline HBV & $10(34)$ & $0(0)$ & 0.083 \\
\hline PBC & $4(14)$ & $1(10)$ & 0.811 \\
\hline ALD & $6(21)$ & $4(40)$ & 0.432 \\
\hline Other & $5(17)$ & $2(20)$ & 0.778 \\
\hline Platelet count $\left(\times 10^{9} / \mathrm{l}\right)$ & $118(39-683)$ & 55 (34-99) & 0.039 \\
\hline AST (IU/I) & $30(17-146)$ & $42(15-120)$ & 0.326 \\
\hline ALT (IU/I) & $24(8-86)$ & $29(9-123)$ & 0.367 \\
\hline Albumin (g/dl) & $3.9(2.7-4.6)$ & $3.5(3.0-4.2)$ & 0.055 \\
\hline T.Bil (mg/dl) & $1.0(0.5-7.5)$ & $2.4(0.4-7.1)$ & 0.014 \\
\hline PT\% & $78(40-95)$ & $64(27-92)$ & 0.004 \\
\hline $\mathrm{LS}(\mathrm{kPa})$ & $7.7(3.5-75.0)$ & $24.9(9.1-75.0)$ & 0.004 \\
\hline Spleen size (cm) & $10.3(6.1-24.0)$ & $13.9(7.7-16.6)$ & 0.050 \\
\hline LSPS & $1.0(0.1-9.5)$ & $5.5(1.2-20.0)$ & $<0.001$ \\
\hline APRI & $1.1(0.1-4.8)$ & $2.4(0.8-4.0)$ & 0.026 \\
\hline FIB-4 & $4.2(0.1-12.0)$ & $8.4(1.2-10.9)$ & 0.044 \\
\hline Platelet-to-spleen ratio & $1092(225-5591)$ & 407 (220-770) & 0.003 \\
\hline
\end{tabular}

Values are expressed as median (range, minimum-maximum) or $\mathrm{n}(\%)$
This investigation had several limitations. It was retrospective in design. Longer follow-up will be required to evaluate the prediction of EV and bleeding EV, especially in patients without high-risk EV in CLD, to overcome a possible selection bias in this study. The relatively small sample size may have influenced the results as well; for example, the LS of alcoholic LD was higher than those of other etiologies $(P<0.001)$, although the number of alcoholic LD patients was too small for any definite conclusions. A further study will be needed to clarify which values of LS (including that for LSPS) can predict EV according to etiology in CLD. Moreover, it was difficult to determine how many patients could be spared endoscopy, could avoid unnecessary endoscopy, or had received unnecessary endoscopy with this study model since approximately $40 \%$ of subjects were excluded due to reasons such as an IQR of greater than $30 \%$. During the study period, 4462 patients received endoscopy at our institution, 251 of whom (5.6\%) had evidence of varices by endoscopy. Fifty-seven of 251 patients with EV (22.7 \%) exhibited high risk EV. As patients with obesity or ascites are not good for candidates for TE, other surrogate fibrosis biomarkers, such as Mac-2-binding protein (Kuno et al. 2013; Umemura et al. 2015), are being considered for these individuals.

Table 5 Performance of noninvasive parameters for identifying high risk EV among 39 patients complicated with EV

\begin{tabular}{|c|c|c|c|c|c|c|c|c|c|}
\hline Characteristic & Cutoff & AUC (95 \% Cl) & $\begin{array}{l}\text { Sensitivity } \\
(\%)\end{array}$ & $\begin{array}{l}\text { Specificity } \\
\text { (\%) }\end{array}$ & $\begin{array}{l}\text { PPV } \\
\text { (\%) }\end{array}$ & $\begin{array}{l}\text { NPV } \\
\text { (\%) }\end{array}$ & $\begin{array}{l}\text { Positive } \\
\text { Likelihood } \\
\text { ratio }\end{array}$ & $\begin{array}{l}\text { Negative } \\
\text { Likelihood } \\
\text { ratio }\end{array}$ & $\begin{array}{l}\text { Accuracy } \\
\text { (\%) }\end{array}$ \\
\hline LSPS & 4.5 & $0.859(0.736-0.981)$ & 70.0 & 86.2 & 63.6 & 89.3 & 5.08 & 0.35 & 82.1 \\
\hline Platelet count ( $\left.\times 10^{9} / 1\right)$ & 100 & $0.833(0.707-0.958)$ & 100.0 & 65.5 & 50.0 & 100.0 & 2.61 & 0.00 & 74.3 \\
\hline Platelet-to-spleen ratio & 990 & $0.817(0.687-0.947)$ & 100.0 & 65.5 & 50.0 & 100.0 & 2.90 & 0.00 & 74.3 \\
\hline $\mathrm{LS}(\mathrm{kPa})$ & 12.0 & $0.807(0.674-0.940)$ & 80.0 & 75.9 & 53.3 & 91.7 & 3.31 & 0.31 & 76.9 \\
\hline APRI & 1.7 & $0.762(0.609-0.915)$ & 80.0 & 72.4 & 50.0 & 91.3 & 2.90 & 0.28 & 74.4 \\
\hline F|B-4 & 5.0 & $0.716(0.529-0.902)$ & 90.0 & 58.6 & 42.9 & 94.4 & 2.18 & 0.17 & 66.7 \\
\hline
\end{tabular}




\section{Conclusion}

This investigation validates LSPS as a potent, noninvasive method for predicting EV and high risk EV in Japanese patients with CLD. Clinicians should recommend those patients with CLD who show higher values of LSPS to undergo further endoscopic examination.

\section{Additional file}

Additional file 1: Figure S1. Frequency of $L S I Q R<30 \%$ vs. that of $L S$ $I Q R \geq 30 \%$ for each etiology. Frequencies of LS IQR $<30 \%$ (dark gray) and those of $L S I Q R \geq 30 \%$ (light gray) according to etiology in all subjects ( $n=835$ ). The frequency of $L S I Q R<30 \%$ for HCV is significantly lower than those of other etiologies. There are no differences among the other etiologies of CLD.

\section{Abbreviations}

APRI: aspartate aminotransferase-to-platelet ratio index; AUC: area under the ROC curve; Cls: confidence intervals; CLD: chronic liver disease; EV: esophageal varices; kPa: kilopascals; LS: liver stiffness; LSPS: LS-spleen diameter-to-platelet ratio risk score; NAFLD: non-alcoholic fatty liver disease; NASH: non-alcoholic steatohepatitis; NPV: negative predictive value; PPV: positive predictive value; ROC: receiver operating characteristic; TE: transient elastography; US: ultrasonography.

\section{Authors' contributions}

SS: study concept and design, acquisition of data, analysis and interpretation of data, statistical analysis. SJ: acquisition of data, analysis and interpretation of data, statistical analysis, drafting of the manuscript, TU: study concept and design, acquisition of data, analysis and interpretation of data, drafting of the manuscript, statistical analysis. TY, NF, YI, MK, AM: acquisition of data. ET: study concept and design, critical revision of the manuscript for important intellectual content, study supervision. All authors read and approved the final manuscript.

\section{Acknowledgements}

The authors thank Ms. Mie Karakida for her secretarial assistance and Mr. Trevor Ralph for his English editorial assistance.

\section{Competing interests}

The authors declare that they have no competing interests.

Received: 8 March 2016 Accepted: 28 June 2016

Published online: 07 July 2016 de Franchis R, Baveno VF (2010) Revising consensus in portal hypertension: report of the Baveno $V$ consensus workshop on methodology of diagnosis and therapy in portal hypertension. J Hepatol 53(4):762-768

Giannini E, Botta F, Borro P, Risso D, Romagnoli P, Fasoli A, Mele MR, Testa E, Mansi C, Savarino V, Testa R (2003) Platelet count/spleen diameter ratio: proposal and validation of a non-invasive parameter to predict the presence of oesophageal varices in patients with liver cirrhosis. Gut 52(8):1200-1205

Kim BK, Han KH, Park JY, Ahn SH, Kim JK, Paik YH, Lee KS, Chon CY, do Kim $Y$ (2010) A liver stiffness measurement-based, noninvasive prediction model for high-risk esophageal varices in B-viral liver cirrhosis. Am J Gastroenterol 105(6):1382-1390

Kleiner DE, Brunt EM, Van Natta M, Behling C, Contos MJ, Cummings OW, Ferrell LD, Liu YC, Torbenson MS, Unalp-Arida A, Yeh M, McCullough AJ, Sanyal AJ (2005) Design and validation of a histological scoring system for nonalcoholic fatty liver disease. Hepatology 41(6):1313-1321

Kuno A, Ikehara Y, Tanaka Y, Ito K, Matsuda A, Sekiya S, Hige S, Sakamoto M, Kage M, Mizokami M, Narimatsu H (2013) A serum "sweet-doughnut" protein facilitates fibrosis evaluation and therapy assessment in patients with viral hepatitis. Sci Rep 3:1065

Sandrin L, Fourquet B, Hasquenoph JM, Yon S, Fournier C, Mal F, Christidis C, Ziol M, Poulet B, Kazemi F, Beaugrand M, Palau R (2003) Transient elastography: a new noninvasive method for assessment of hepatic fibrosis. Ultrasound Med Biol 29(12):1705-1713

Shibata S, Umemura T, Yamazaki T, Fujimori N, Ichikawa Y, Kimura T, Joshita S, Komatsu M, Matsumoto A, Tanaka E (2015) Liver stiffness-spleen size-toplatelet ratio risk score identifies esophageal varices in japanese patients with chronic hepatitis C. Hepatol Res

Sterling RK, Lissen E, Clumeck N, Sola R, Correa MC, Montaner J, Sulkowski S, Torriani FJ, Dieterich DT, Thomas DL, Messinger D, Nelson M, Investigators AC (2006) Development of a simple noninvasive index to predict significant fibrosis in patients with HIV/HCV coinfection. Hepatology 43(6):1317-1325

Umemura T, Zen Y, Hamano H, Kawa S, Nakanuma Y, Kiyosawa K (2007) Immunoglobin G4-hepatopathy: association of immunoglobin G4-bearing plasma cells in liver with autoimmune pancreatitis. Hepatology 46(2):463-471

Umemura T, Tanaka E, Kiyosawa K, Kumada H (2008) Mortality secondary to fulminant hepatic failure in patients with prior resolution of hepatitis $B$ virus infection in Japan. Clin Infect Dis 47(5):e52-e56

Umemura T, Joshita S, Ichijo T, Yoshizawa K, Katsuyama Y, Tanaka E, Ota M (2012) Human leukocyte antigen class II molecules confer both susceptibility and progression in Japanese patients with primary biliary cirrhosis. Hepatology 55(2):506-511

Umemura T, Joshita S, Sekiguchi T, Usami Y, Shibata S, Kimura T, Komatsu M, Matsumoto A, Ota M, Tanaka E (2015) Serum Wisteria floribunda agglutinin-positive Mac-2-Binding protein level predicts liver fibrosis and prognosis in primary biliary cirrhosis. Am J Gastroenterol 110(6):857-864

Wai CT, Greenson JK, Fontana RJ, Kalbfleisch JD, Marrero JA, Conjeevaram HS, Lok AS (2003) A simple noninvasive index can predict both significant fibrosis and cirrhosis in patients with chronic hepatitis C. Hepatology 38(2):518-526

\section{Submit your manuscript to a SpringerOpen ${ }^{\odot}$ journal and benefit from:}

- Convenient online submission

- Rigorous peer review

- Immediate publication on acceptance

- Open access: articles freely available online

- High visibility within the field

- Retaining the copyright to your article

Submit your next manuscript at springeropen.com 